\title{
Exact solutions for near-wall turbulence theory
}

\author{
S. Nazarenko \\ Mathematics Institute, University of Warwick, Coventry CV4 7AL, United Kingdom \\ Received 1 September 1999; received in revised form 18 November 1999; accepted 25 November 1999 \\ Communicated by A.P. Fordy
}

\begin{abstract}
Using the 2D case as a simple example, we outline an analytical approach to the near wall turbulence outside of the viscous sublayer. Our theory combines the Reynolds averaged mean-flow equation nonlinearly coupled to the RDT equations for turbulence with a weak small-scale forcing. Such an external forcing models the dilute vortex debris propagating away from the wall as a result of intermittent bursts accompanying the breakdown of the coherent vortices in the viscous sublayer. We show that the Log law of the wall exists as an exact analytical solution in our model if the starting turbulent vorticity is statistically homogeneous in space and shortly correlated in time. (C) 2000 Elsevier Science B.V. All rights reserved.
\end{abstract}

PACS: $47.27 . \mathrm{Nz} ; 4$ 77.27.Eq

Often, most interesting physical phenomena are too hard to be explained rigorously in all their generality. One of them, the near-wall turbulence, has been a subject of lively discussion in literature over several past years. The focus of this discussion has been on the question whether turbulence possesses the complete similarity property, a classical picture of Von Karman [1] and Prandtl [2] predicting the Log law of the wall, or it is characterized by an incomplete similarity and power law mean profiles as suggested by Barenblatt et al. [3-5]. Scaling theories, even in their refined form [3-6], proved to be insufficient for resolving such a controversy because, being phenomenological in nature, they involve adjustable parameters and even contradicting theories can often fit the same experimental [7-9] or numerical data [10]. On the other hand, understanding of physical mechanisms is usually helped by exact solutions obtained directly from the Navier-
Stokes equations for special idealized situations and serving as reference points for more general theory. It is the lack of such exact results that obscures understanding of the near-wall turbulence, and the aim of the present letter is to obtain some rigorous analytical solutions by combining the Rapid Distortion theory (RDT) and the averaged Reynolds stress description of the mean flow. The idea to couple RDT and the mean flow equations was suggested for turbulent shear flows by Moffatt [11]. New element introduced in the present Letter is an external forcing in the RDT equations that allows to obtain solutions corresponding to statistically stationary turbulence. In real near-wall turbulence the turbulence forcing outside of the viscous sublayer is provided by continuous supply of starting vorticity propagating into the outer regions as a result of intermittent bursts of coherent structures generated inside the viscous sublayer. In this Letter we will only consider the sim- 
plest case of 2D shear flow turbulence leaving the detailed study of a more realistic 3D case for separate publication $[12,13]$.

Let us write the 2D Navier-Stokes equations formulated in terms of vorticity $\omega$ as

$\partial_{t} \omega+\boldsymbol{u} \cdot \nabla \omega=\sigma+\nu \nabla^{2} \omega$,

( $\sigma$ is a random external forcing) and let us assume that the velocity field $\boldsymbol{u}$ consists of a large-scale shear flow $U(y) e_{x}$ and small-scale turbulence $\tilde{\boldsymbol{u}}$. In spirit of RDT, let us assume turbulence to be weak compared to the mean flow, $|\tilde{\omega}|=\left|\partial_{x} u_{y}-\partial_{y} u_{x}\right| \ll$ $|\Omega|=\left|\partial_{y} U\right|$, and let us linearize the Navier-Stokes equations,

$\partial_{t} \tilde{\boldsymbol{\omega}}+U \partial_{x} \tilde{\boldsymbol{\omega}}+\tilde{u}_{2} \Omega^{\prime}(y)=\sigma+\nu \nabla^{2} \tilde{\boldsymbol{\omega}}$,

We assumed that the forcing $\sigma$ has a scale $l$ which is much less than characteristic scale of the mean flow $L$, i.e. $\epsilon=l / L \ll 1$. This implies that turbulence is small-scale and one can neglect the term $\tilde{u}_{2} \Omega^{\prime}$ and use a WKB description using Gabor transform, which is defined as

$$
\begin{aligned}
\hat{\boldsymbol{\omega}}(y, \boldsymbol{k}, t)= & \int f\left(\boldsymbol{\epsilon}^{*}\left|y-y_{0}\right|\right) \mathrm{e}^{-i p x_{0}+i q\left(y-y_{0}\right)} \\
& \times \tilde{\boldsymbol{\omega}}\left(\boldsymbol{x}_{0}, t\right) d \boldsymbol{x}_{0},
\end{aligned}
$$

where $\boldsymbol{k}=(p, q)$ is the wavevector, $k \sim 2 \pi / l, 1 \gg$ $\epsilon^{*} \gg \epsilon$, and $f(x)$ is a rapidly decreasing at infinity function, for example $\mathrm{e}^{-x^{2}}$. Note that the WKB equations can be also obtained using Wigner function $[14,15]$, although using Gabor transform allows a simpler derivation [16] (1),

$$
\begin{aligned}
\partial_{t} \hat{\boldsymbol{\omega}} & +\int f\left(\epsilon^{*}\left|y-y_{0}\right|\right) \mathrm{e}^{-i p x_{0}+i q\left(y-y_{0}\right)} \\
& \times U\left(y_{0}\right) \partial_{x 0} \tilde{\boldsymbol{\omega}}\left(\boldsymbol{x}_{0}\right) d \boldsymbol{x}_{0}=\hat{\boldsymbol{\sigma}}-\nu k^{2} \hat{\boldsymbol{\omega}} .
\end{aligned}
$$

Because of the decreasing kernel $f$, the main contribution into the integral here comes from $\left|y-y_{0}\right| \sim$ $1 / \epsilon^{*}$. Therefore, one can Taylor expand function $U\left(y_{0}\right)$ which varies significantly only at the large scale $1 / \epsilon \gg 1 / \epsilon^{*}$. Neglecting the quadratic terms in this Taylor expansion, which are small as $\left(\epsilon / \epsilon^{*}\right)^{2}$, we have

$$
\begin{aligned}
\partial_{t} \hat{\boldsymbol{\omega}} & +\int f\left(\boldsymbol{\epsilon}^{*}\left|y-y_{0}\right|\right) \mathrm{e}^{-i p x_{0}+i q\left(y-y_{0}\right)} \\
& \times U(y) \partial_{x_{0}} \tilde{\boldsymbol{\omega}}\left(\boldsymbol{x}_{0}\right) d \boldsymbol{x}_{0} \\
& +\int f\left(\boldsymbol{\epsilon}^{*}\left|y-y_{0}\right|\right) \mathrm{e}^{-i p x_{0}+i q\left(y-y_{0}\right)}\left(y_{0}-y\right) \\
& \times U^{\prime}(y) \partial_{x_{0}} \tilde{\boldsymbol{\omega}}\left(\boldsymbol{x}_{0}\right) d \boldsymbol{x}_{0}=\hat{\boldsymbol{\sigma}}-\nu k^{2} \hat{\boldsymbol{\omega}} .
\end{aligned}
$$

Integrating by parts in both integrals and changing $\left(y_{0}-y\right) \rightarrow i \partial_{q}$ in the second integral we have

$\partial_{t} \hat{\omega}+i p \hat{\omega} U-U^{\prime} p \partial_{q} \hat{\omega}=\hat{\sigma}-\nu k^{2} \hat{\omega}$.

This is a linear first-order ODE providing RDT description of turbulence in two dimensions. This equation can be solved exactly for arbitrary forcing $\sigma$ and viscosity $\nu$. However, for the sake of clarity and brevity we are not going to do so but simply mention that viscosity, if small, does not affect the mean flow dynamics and can be neglected. This is because the high-wavenumber part of the turbulent spectrum contains almost no energy in 2D. This property is not valid for 3D turbulence for which RDT predicts growth of the turbulent energy because of the vortex stretching. Hereafter we will ignore viscosity, which will make our analysis much simpler than in 3D case (which will be considered in Refs. [12,13]).

We obtain the mean flow equation in a standard way by averaging of the Navier-Stokes,

$$
\begin{aligned}
& \partial_{t} U=-\partial_{y} \tau-\partial_{x} P, \\
& \tau=\left\langle u_{1} u_{2}\right\rangle,
\end{aligned}
$$

where, as usually, the pressure gradient $\partial_{x} P$ is constant. We ignored viscosity in (7) because we we will be only interested to describe the mean profile far from the viscous sublayer. The easiest averaging procedure that allows to write the mean flow and 
turbulence equations in a closed form is combination of the ensemble averaging (denoted by overbar) and a space filtering,

$$
\begin{aligned}
\langle a\rangle(y, t)= & \lim _{R \rightarrow \infty} \frac{1}{2 R} \int_{-R}^{R} d x_{0} \int_{-\infty}^{\infty} d y_{0} \\
& \times f^{2}\left(\boldsymbol{\epsilon}^{*}\left|y-y_{0}\right|\right) \bar{a}\left(\boldsymbol{x}_{0}, t\right),
\end{aligned}
$$

where $f$ is the same function as in the Gabor transform (3), and, therefore, the space filtering in $y$ is over a length which is intermediate between the small scale $l$ and the large scale $L$. In this case,

$$
\begin{aligned}
\tau(y) & =\frac{1}{(2 \pi)^{2}} \int \overline{\hat{u}_{1}(y, \boldsymbol{k}) \hat{u}_{2}(y,-\boldsymbol{k})} d \boldsymbol{k} \\
& =\frac{1}{(2 \pi)^{2}} \int \frac{p q}{k^{4}} \overline{\hat{\omega}(y, \boldsymbol{k}) \hat{\omega}(y,-\boldsymbol{k})} d \boldsymbol{k} .
\end{aligned}
$$

This expression for the averaged Reynolds stress was obtained before in Refs. [14,15]. Let us multiply Eq. (6) by $\hat{\omega}^{*}(y, \boldsymbol{k})=\hat{\omega}(y,-\boldsymbol{k})$ and average the result assuming a stationary state, $\partial_{t}\left\langle\omega \omega^{*}\right\rangle=\partial_{t} U=0$. Neglecting viscosity, we have

$-U^{\prime} p \partial_{q}\left\langle\omega \omega^{*}\right\rangle=F$.

where $F=2 \operatorname{Re}\left(\overline{\hat{\sigma} \hat{\omega}^{*}}\right)$. Integrating in (9) by parts with respect to $q$ and using (10), we have

$\tau(y)=-\frac{\lambda}{U^{\prime}}$,

with

$\lambda=\frac{1}{2(2 \pi)^{2}} \int \frac{F}{k^{2}} d \boldsymbol{k}$.

Note that $\lambda>0$ and, therefore, the turbulent momentum flux is always in the same direction as the viscous one. On the other hand, the $y$-derivative of the turbulent flux has opposite sign to the one of the viscous term $\nu U^{\prime \prime}$ (because $\operatorname{sign} U^{\prime}=-\operatorname{sign} U^{\prime \prime}$ ) and, therefore, the turbulent friction is negative in $2 \mathrm{D}$ case. Such a phenomenon is an artifact of the $2 \mathrm{D}$ geometry of the problem which is closely related to the fact of negative eddy diffusivity and inverse energy cascade in $2 \mathrm{D}$. The turbulent friction will be shown to be positive in $3 \mathrm{D}$ [12].
Substituting Eq. (11) into the stationary version of the mean flow equation Eq. (6) and integrating over $y$ we have

$\lambda / U^{\prime}=\partial_{x} P y$.

The integration constant here is chosen to be zero which corresponds to fixing the coordinate origin. In the important special case of short correlated in time forcing $\sigma$ the second and the third terms in the RHS of Eq. (6) are much less than the first one so that $\hat{\omega}(\boldsymbol{k}, t) \approx \int_{0}^{\infty} \hat{\boldsymbol{\sigma}}\left(\boldsymbol{k}, t-t_{1}\right) d t_{1}$ and

$F(\boldsymbol{k})=2 \operatorname{Re} \int_{-\infty}^{0} \overline{\hat{\sigma}(\boldsymbol{k}, t) \hat{\sigma}(-\boldsymbol{k}, 0)} d t$

If, in addition, forcing $\sigma$ is statistically uniform in space (i.e. $\hat{\sigma}$ is independent of $y$ ) then $F$ is independent of $y$ and $\lambda=$ const. In this case one can further integrate Eq. (13) and obtain

$U=\frac{\lambda}{\partial_{x} P} \log y$

As we see, the celebrated Log law of the wall can be directly derived from the Navier-Stokes equations with an external turbulence forcing assuming that this forcing is (i) weak, (ii) small-scale, (iii) short correlated in time and (iv) statistically homogeneous in space. Note that the first two assumptions are essential for validity of our model because they allow to use RDT and the scale separation technique. These assumptions about the turbulence forcing seem to be realistic because in reality the starting vorticity is generated in a thin viscous sublayer which makes it small-scale. It further propagates away from the wall and gets diluted in a large (compared to the viscous sublayer) volume which makes it weak. On the other hand, properties (iii) and (iv) are essential for existence of the logarithmic profile, but they are not important for validity of the RDT-type description. For example, if turbulence forcing is a power law function $\hat{\sigma} \sim y^{a}$ then the Log law is replaced by a power law profile of the mean velocity.

Finally, let us use our theory to calculate the one-dimensional spectrum $\phi_{11}(p, y)=(1 / 4 \pi)$ $\int \hat{u}_{1} \hat{u}_{1}^{*} d q$ which is frequently measured in experiments. Because we are dealing with Gabor transforms of real functions, it is enough to consider only 
half of the wavenumber space $q<0$ and double the result; we have

$$
\begin{gathered}
\phi_{11}(p, y)=\frac{1}{2 \pi} \int_{-\infty}^{0} \hat{u}_{1} \hat{u}_{1}^{*} d q=\frac{1}{2 \pi} \int_{-\infty}^{0} \frac{q^{2}}{k^{4}} \hat{\omega} \hat{\omega}^{*} d q \\
=-\frac{1}{2 \pi} \int_{-\infty}^{0}\left(\int_{-\infty}^{q} \frac{q_{1}^{2} d q_{1}}{\left(p^{2}+q_{1}^{2}\right)^{2}}\right) \partial_{q}\left(\hat{\omega} \hat{\omega}^{*}\right) d q \\
=\frac{1}{2 \pi U^{\prime} p} \int_{-\infty}^{0}\left(\int_{-\infty}^{q} \frac{q_{1}^{2} d q_{1}}{\left(p^{2}+q_{1}^{2}\right)^{2}}\right) F(p, q) d q
\end{gathered}
$$

In the range $p \ll k_{*}$ (where $k_{*}$ is a characteristic wavenumber of the forcing $F(k))$ we have

$$
\begin{aligned}
\phi_{11}(p, y) & \approx-\frac{1}{2 \pi U^{\prime} p} \int_{-\infty}^{0} \frac{F(0, q)}{q} d q \\
& =\frac{1}{4 \pi U^{\prime} p} \int_{-\infty}^{\infty} \frac{F(0, q)}{|q|} d q
\end{aligned}
$$

Taking into account Eq. (13) one can write

$\phi_{11}(p, y)=\frac{C u_{*}^{2} y}{H} \frac{1}{p}$,

where $C$ is a non-dimensionless constant,

$C=\frac{1}{2 \pi \lambda} \int_{-\infty}^{\infty} \frac{F(0, q)}{|q|} d q$.

Here, we took into account $\partial_{x} P=2 u_{*}^{2} / H$ where $u_{*}$ is the friction velocity and $H$ is the channel width. One can see that for $p \ll k_{*}$ the spectrum becomes universal in that its exponent does not depend on the particular form of the forcing function. Universal $p^{-1}$ spectra are indeed observed experimentally in the low wavenumber range although their $y$ dependence is different from the one in Eq. (18) [17].

In the next paper [12] we will consider the 3D case which involves more complicated algebra but it is more realistic in that the turbulent perturbations are amplified by the mean flow rather than suppressed as in the $2 \mathrm{D}$ case. Also, the 3D theory predicts positive turbulent friction, as it has to be in real shear flows. We will show however that in more realistic 3D model the conditions of realizability of the Log law remain essentially the same as for the simple 2D case considered in this Letter. This indicates that starting vorticity (generated in the sublayer) is an important quantity that affects the shear flow turbulence and the mean velocity profile and that this object has to be examined experimentally and using the numerical data. In particular, if vorticity gets spread nearly uniformly by the intermittent sublayer bursts and if its correlation is short, one should expect universal Log law of the wall (which is insensitive to the spectral shape of the starting vorticity). We will also show in the nex paper that viscosity and anisotropy play an essential role in 3D shear flow turbulence (in contrast with 2D), in particular in determining the turbulent stresses and spectra. This arises because, unlike in $2 \mathrm{D}$, the $3 \mathrm{D}$ perturbations grow and a finite viscosity is needed to determine their maximum amplitude and regularize the intgrals defining the turbulent stresses and spectra. Finally, 3D geometry brings about curvature effects which leed to growing waves not described by RDT [18]. These waves play an important role very close to the wall as they may contribute to the vorticity bursting process; their study, therefore, is important to describe the full cycle of self-supporting turbulence re-generation.

\section{References}

[1] Th. von Karman, Mechanische Aehnlichkeit und Turbulenz, Nach. Ges. Wiss. Göttingen Math-Phys. Klasse, 1932, pp. 58-76.

[2] L. Prandtl, Zur turbulenten Stroemung in Rohren und laengs Platten, Ergeb. Aerodyn. Versuch., Series 4, Göttingen, 1932.

[3] G.I. Barenblatt, J. Fluid Mech. 248 (1993) 513.

[4] G.I. Barenblatt, A.J. Chorin, Proc. Nat. Acad. Sci. USA 93 (1996) 6749.

[5] G.I. Barenblatt, A.J. Chorin, V.M. Prostokishin, Proc. Nat. Acad. Sci. USA 94 (1997) 773.

[6] W. George, L. Castillo, M. Wosnik, TAM Report No 872, UILU-ENG-97-6033, University of Illinois at UrbanaChampain, November 1997.

[7] J. Nikuradze, Forsch. Arb. Ing.-Wes. No 356 (1932).

[8] M.V. Zagarola, A.J. Smits, Phys. Rev. Lett. 78 (1997) 239.

[9] M.V. Zagarola, A.E. Perry, A.J. Smits, Phys. Fluids 9 (1997) 2094.

[10] J. Kim, R. Moser, J. Fluid Mech. 177 (1987) 133.

[11] H.K. Moffatt, The interaction of turbulence with a strong wind shear, in: A.M. Yaglom, V.I. Tatarsky (Eds.), Proc. URSI-IUGG International Colloquim on Atmospheric turbu- 
lence and radio wave propagation, Moscow, June 1965 Nauka Moscow, 1967.

[12] S. Nazarenko, N.K.-R. Kevlahan, B. Dubrulle, Nonlinear RDT theory of near-wall turbulence, to appear in Physica D (1999).

[13] B. Dubrulle, J.-P. Laval, S. Nazarenko, N.K.-R. Kevlahan, Derivation of equilibrium profiles in plane parallel flows using a dynamic subgrid-scale model, submitted to Phys. Fluids (1999).
[14] A.I. Dyachenko, S.V. Nazarenko, V.E. Zakharov, Phys. Lett. A 165 (1992) 330.

[15] B. Dubrulle, S. Nazarenko, Physica D 110 (1997) 123.

[16] S.V. Nazarenko, N. Kevlahan, B. Dubrulle, J. Fluid Mech. 390 (1999) 325.

[17] A.E. Perry, S. Henbest, M.S. Chong, J. Fluid Mech. 165 (1986) 163.

[18] A.M. Savill, Ann. Rev. Fluid Mech. 19 (1987) 537. 